\title{
A Simplified Analysis of an Electrical Network with Resistive and/or Reactive Elements
}

\author{
Dr. Dolores De Groff
}

College of Engineering, Florida Atlantic University, Boca Raton, FI. 33431

degroff@fau.edu

\begin{abstract}
Typically star-delta transformation is adopted in determining the equivalent impedance between two terminals of an electrical network [2]. Shown relevantly in [1] is a simplified approach, less time-consuming to determine the equivalent resistance between two terminals of an electrical circuit made of resistive elements (of equal values) and, hence the resultant currents in the ciurcuit are determined. This concept is extended and generalized in this study to a class of networks with unequal resistances and/or reactances to determine the equivalent impedance between two nodes. This generalized approach is more comprehensive and universal in analyzing electrical network with complex impedances (regardless of their values). It is applicable to both contexts of circuits with pure resistive elements (as in d.c. analysis) as well as with complex impedances encountered in a.c. analysis.
\end{abstract}

\section{Indexing terms/Keywords}

Bridge circuits, null, wye-delta transformation, equivalent resistance, equivalent impedance, complex network, open circuit, node elimination

\section{Academic Discipline And Sub-Disciplines}

Engineering

\section{SUBJECT CLASSIFICATION}

Network Analysis Theory

\section{TYPE (METHOD/APPROACH)}

Computational research

\section{Council for Innovative Research}

Peer Review Research Publishing System

Journal: INTERNATIONAL JOURNAL OF COMPUTERS \& TECHNOLOGY

Vol 12, No. 6

editor@cirworld.com

www.cirworld.com, www.ijctonline.com 


\section{INTRODUCTION}

Certain electrical circuits (such as those found in power system applications) can easily have hundreds of nodes. However, only a few of these nodes have sources connected to them. If the nodes that do not have sources are removed (on ad hoc basis), the circuit analysis of the system gets significantly simplified. Such node elimination in complicated systems can be done using matrix operations (by computer); however, it can also be done by hand calculation, depending on the number of nodes. A common method (given in most network analysis textbooks [2]) to solve by hand is to use wye-delta transformations and then series-parallel combinations as necessary. That is, in the contexts of analyzing such networks, (both d.c. and/or a.c.) often star-delta transformation plus necessary series and parallel simplification of the elements are involved to find the circuit equivalent and determine relevant node voltages and loop-currents.

Pertinent to such analysis Aneja and Kumar [1] have recently indicated a simplified alternative method (in lieu of star-delta transformation). The approach is shown to be less time-consuming and simplified vis-à-vis complex topology of electrical network. The study in [1] is confined to resistive elements, and such elements are also assumed to be all equal.

The present study is indicated to perform a simplified analysis in more comprehensive electrical networks that may contain both resistive and/or reactive elements; also, no assumption is needed on the elements being all of identical values, except that a certain ratio relation has to be maintained between elements in designated arms. The proposed method is also simple and less time-consuming; but, has a broader scope to include reactive elements and as such, is applicable to both d.c. and a.c. networks as well.

\section{STATEMENT OF THE PROBLEM: PROPOSED METHODOLOGY}

To illustrate the scope of the study and demonstrate the underlying efficacy, the following explicit configurations of circuits are considered:

- $\quad$ Circuits with unequal resistance values

- $\quad$ Circuits configured with equal-valued complex impedances

- $\quad$ Circuits with unequal impedance values

\section{ALGORITHM}

The shortcut procedure given in [1] refers to finding the equivalent resistance for examples across terminals $A$ and $B$ in Fig. 1 of [1] assuming $R_{1}=R_{2}=R_{3}=R_{4}=R_{5}=4 \square$ (all being equal). Relevant steps are as follows:

- Keep intact all the branches connected to the terminals across which the equivalent resistance is calculated

- Search for that resistive branch in the circuit that would be a null detection branch of the four-arm bridge

- Open the null-detection branch obtained in the step above.

Fig. 1 of $[1]$ is reproduced here for easy reference. Hence, the equivalent resistance between A and B (in Fig. 1) of [1] is obtained; as well as the loop currents being deduced.

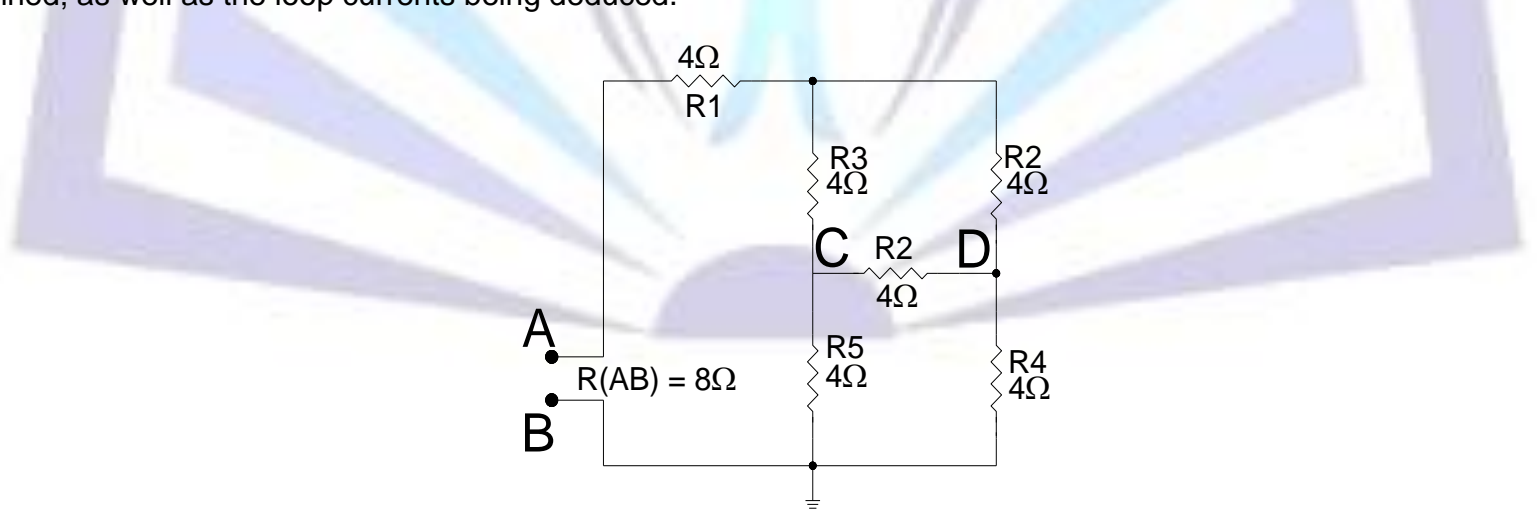

Fig. 1: Figure 1 of [1]

\subsection{Unequal Resistive Network: Present Method}

As stated earlier, in Fig. 1 of [1], the resistive elements considered are all of the same value. However, ahould these values differ, as shown in Fig. 2 below, a relevant modified procedure is indicated. The traditional method of finding equivalent resistance is first illustrated using wye-to-delta transformation. As an example, In the following figure (Fig. 2), wye-to-delta transformations are used to find the resistance between terminals $A$ and $B$. 


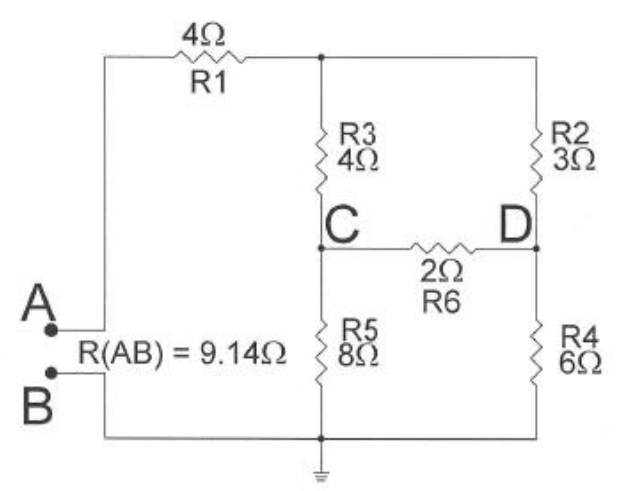

Fig. 2: Unequal Resistive Network Following the Condition of Balance

Figure 2 can be redrawn using the wye-to-delta transformation as illustrated in Fig. 3. Relevant relations are given here for a circuit with components specified as follows:

$R_{\mathrm{a}}=\frac{R_{Y 1} R_{Y 2}+R_{Y 2} R_{Y 3}+R_{Y 3} R_{Y 1}}{R_{Y 1}}, R_{b}=\frac{R_{Y 1} R_{Y 2}+R_{Y 2} R_{Y 3}+R_{Y 3} R_{Y 1}}{R_{Y 2}}, R_{c}=\frac{R_{Y 1} R_{Y 2}+R_{Y 2} R_{Y 3}+R_{Y 3} R_{Y 1}}{R_{Y 3}}$
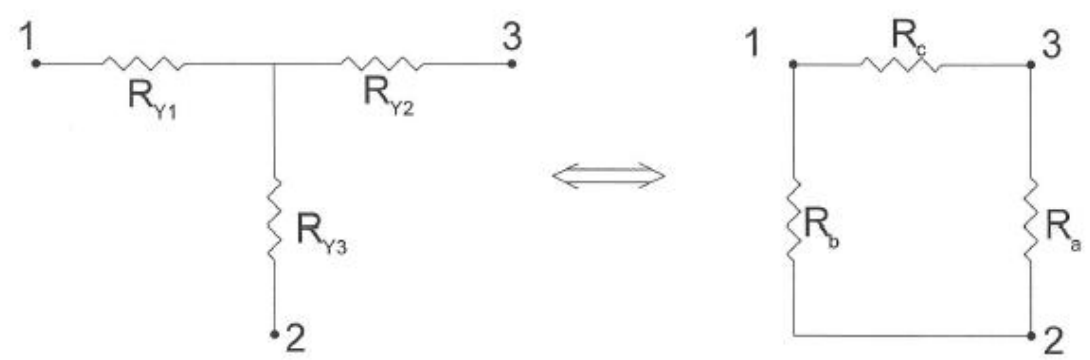

Fig 3: Wye-Delta Transformation of Fig. 2

Thus, considering the circuit shown (Fig. 2) and applying the above relations (1), the resulting numerical values are indicated as in Fig. 4:
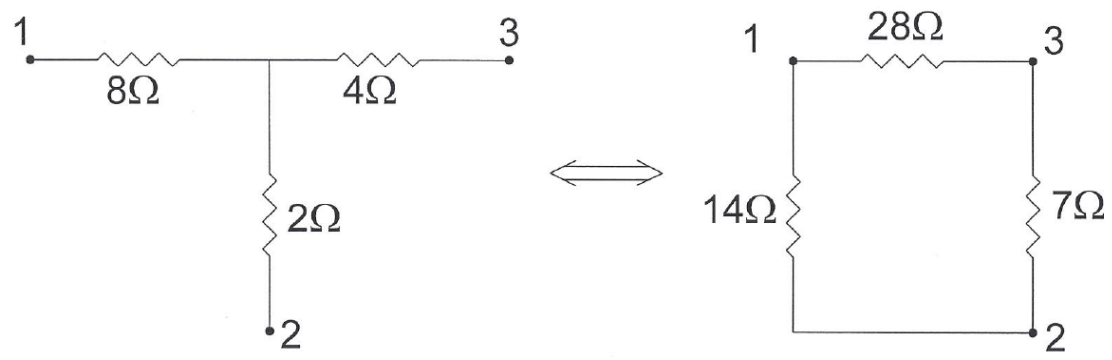

Fig 4: Wye-Delta Transformation for Fig. 2 with numerical values 
Suppose Fig. 2 is redrawn as in Fig. 5:

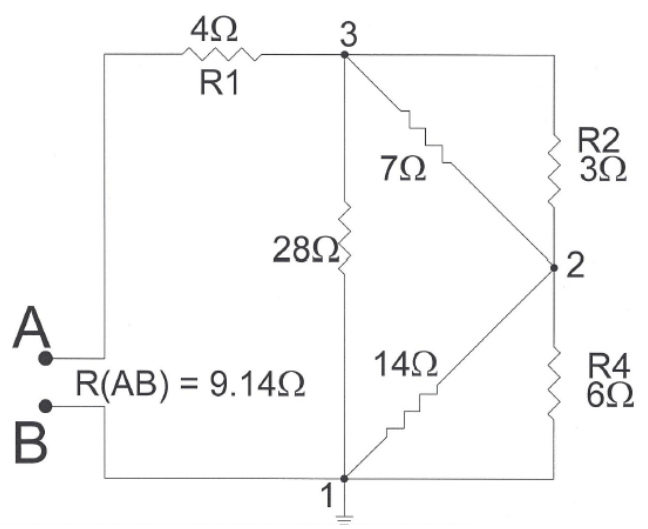

Fig 5: Circuit Redrawn Using Wye-Delta Transformation

In Fig. 5, the equivalent resistance can be obtained as: $R_{e q}=4+[28 \|((14 \| 6)+(7 \| 3))]=9.14 \Omega$. However, the underlying computations can be simplified by using the modified method of [1] as described below.

The resistor across terminals $C$ and $D$ in Fig. 2 will have a negligible effect if there is a condition of balance existing in the circuit. The proposed method involves searching for values of R2, R3, R4, R5 and determing by inspection whether a condition of balance exists. That is,

$$
\frac{R 3}{R 5}=\frac{R 2}{R 4}
$$

In Fig. 2 note that any current flowing through resistor R3 also flows throught resistor R5. Any current flowing through R2 also flows throught R4. There is no current flowing through R6 and no voltage drop across R6. R6 has no effect on the overall network resistance.

By inspection, for an equal resistive network the balance equation will hold. In addition, there are an infinite number of other possibilities where the balance equation is satisfied and the new shortcut method due to [1] can be used for analysis in such cases. Because the balance relation (2) holds for the circuit in Fig. 2, one can solve for the equivalent resistance between terminals $A$ and $B$ by open-circuiting the branch between terminals $C$ and $D$ since it is a null detection branch of the four-arm bridge. Relevant procedure is illustrated in Fig. 6.
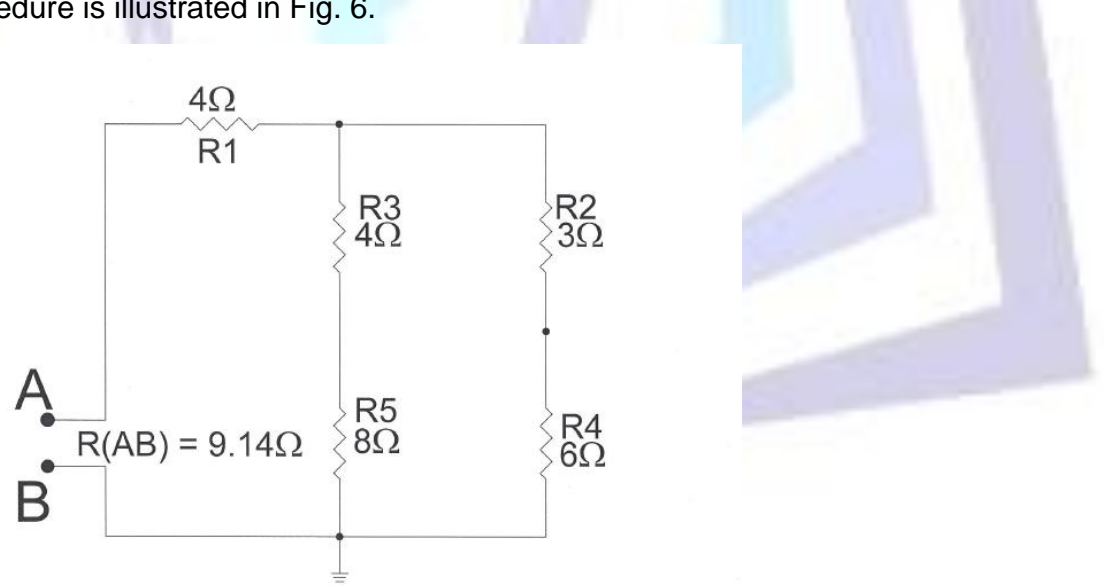

Fig 6: Circuit Redrawn Without Null Branch

A simple calulation gives $R(A B)=4+12 \| 9=9.14 \square$. Note that this is the same result as from the wye-delta transformation approach. Note that the value of the null detection branch resistance does not influence the overall network resistance.

\subsection{Equivalent Impedance AC Network}

It is shown here that the shortcut method can also be applied to determine the equivalent a.c. impedance. As an example consider the proposed circuit in Fig. 7 with complex impledance values: 


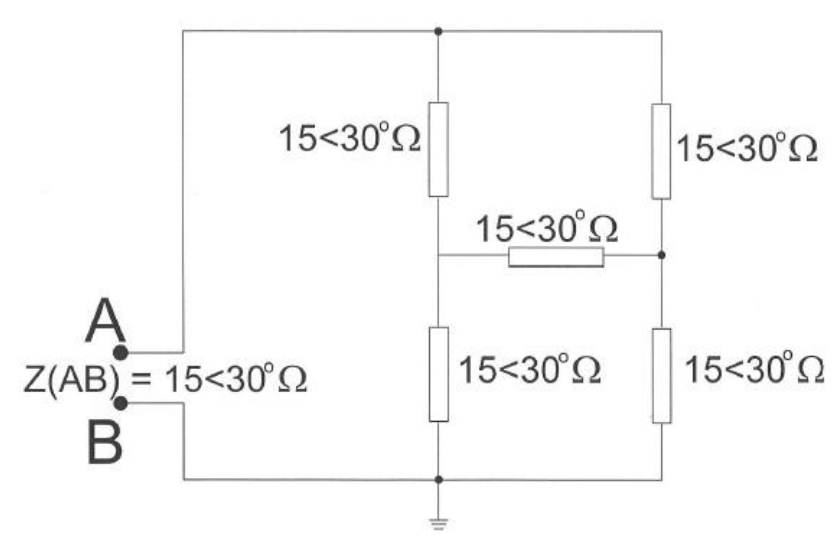

Fig 7: Equivalent Impedance AC Network

Applying wye-delta transformations and noticing that all three impedances connected in wye are equal, it follows that all three impedances connected in delta must be equal and the value of each delta-connected impedance must be three times the value of each wye-connected impedance. This is shown in the figure 8.

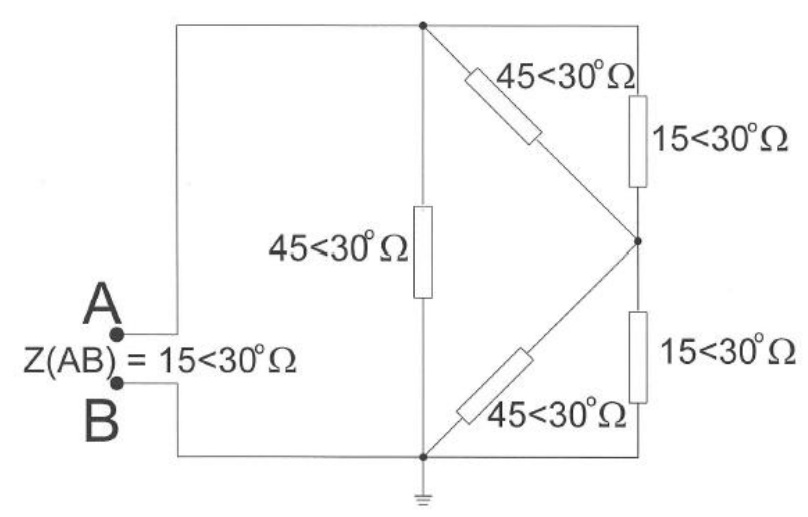

Fig 8: Equivalent Impedance AC Network wye-delta Transformation

Calulation of impedance $Z(A B)$ between terminals $A$ and $B$ in Fig. 8 is given by::

$Z(A B)=\left\{45<30^{\circ} \|\left[\left(15<30^{\circ} \| 45<30^{\circ}\right)+\left(15<30^{\circ} \| 45<30^{\circ}\right)\right]\right\}=15<30^{\circ}$. By not including the null detection branch of the four-arm bridge as per the proposed notion, Fig. 7 simplifies to Fig. 9:

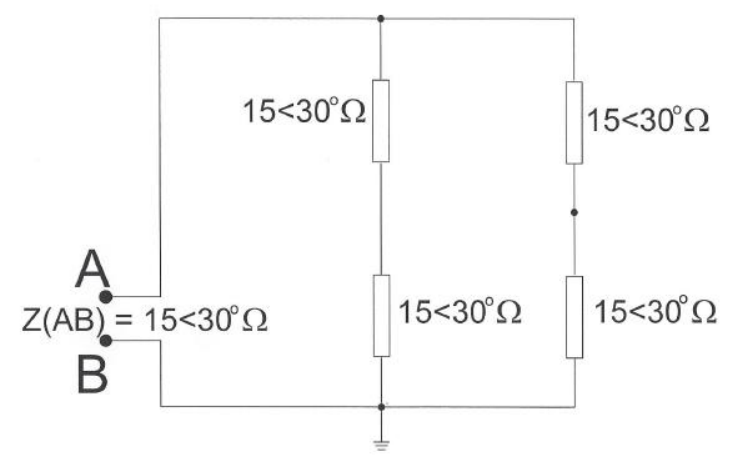

Fig 9: Equivalent Impedance AC Network Using Shortcut Circuit 
By inspection, the equivalent impedance for the circuit in Fig. 8 above is $Z(A B)=30<30^{\circ} \| 30<30^{\circ}=15<30^{\circ}$. Notice that this result is the same as that of the result using the wye-delta transformation approach. Thus the shortcut method has been shown to be successful in equivalent impedance networks as well.

\subsection{Unequal Impedance AC Network}

It is further shown here that the proposed method can also be applied to an unequal impedance a.c. network (as long as certain conditions of balance hold). As an example, consider the following circuit (Fig. 10) :

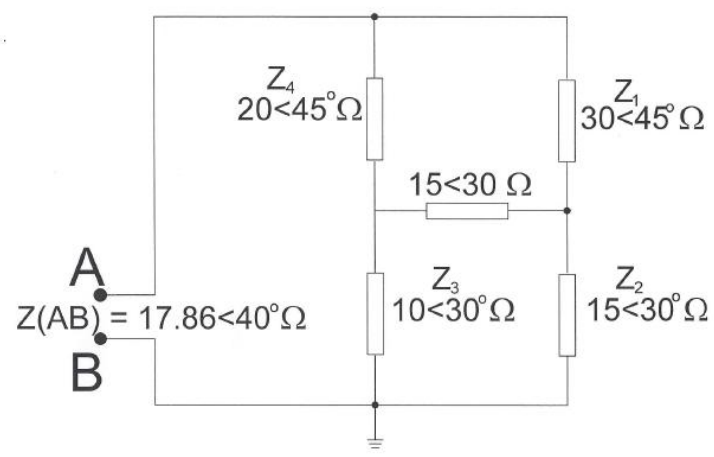

Fig 10: Unequal Impedance AC Network

The circuit in Fig. 10 can be simplified through a wye-delta transformation similar to that discussed previously, and the equivalent impedance is caluculated. For the network shown in Fig. 10, the calculations yield: $Z(A B)=17.86<40^{\circ}$. However, it can be seen by inspection that a balance condition, namely,

$$
\frac{Z_{1}}{Z_{2}}=\frac{Z_{4}}{Z_{3}}
$$

exists in the circuit of Fig. 10. If the circuit is at balance, equation (3) holds and the shortcut procedure can be adopted. Note that for an AC circuit, $Z_{1}=\left|Z_{1}\right|<\square_{1}, Z_{2}=\left|Z_{2}\right|<\square_{2}, Z_{3}=\left|Z_{3}\right|<\square_{3}$, and $Z_{4}=\left|Z_{4}\right|<\square 4$, Then from equation (3), $\left(\left|Z_{1}\right|<\alpha_{1}\right)\left(\left|Z_{3}\right|<\alpha_{3}\right)=\left(\left|Z_{2}\right|<\alpha_{2}\right)\left(\left|Z_{4}\right|<\alpha_{4}\right)$, which shows there are two conditions to be satisfied simultaneoously for balance in an A.C. bridge circuit so that the shortcut method can be used and the null branch has no effect:

$$
\left|Z_{1}\right|\left|Z_{3}\right|=\left|Z_{2}\right|\left|Z_{4}\right| \text { and }\left(\alpha_{1}+\alpha_{3}\right)=\left(\alpha_{2}+\alpha_{4}\right)
$$

Because the balance condition is satisfied for the circuit of Fig. 10, the circuit can be redrawn will the null branch neglected as shown in Fig. 11 towards simplification.

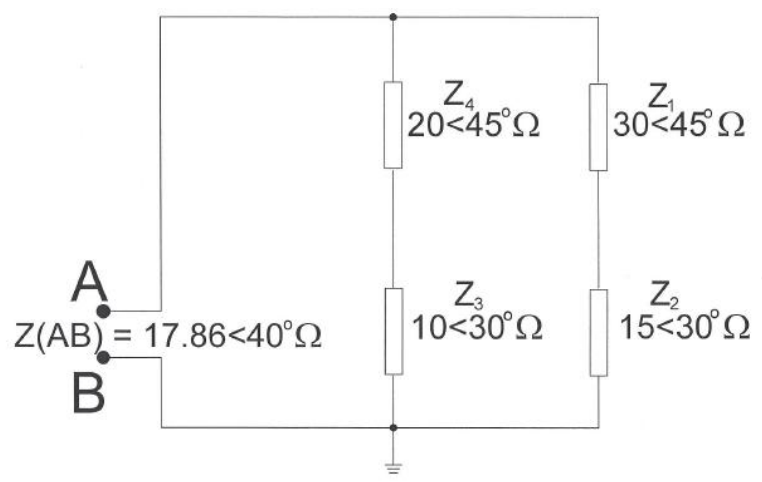

Fig 11: Unequal Impedance AC Network Without Null Branch

From the circuit of Fig. 10, $\mathrm{Z}(\mathrm{AB})=\left[\left(10<30^{\circ}+20<45^{\circ}\right) \|\left(15<30^{\circ}+30<45^{\circ}\right)\right]=17.86<40^{\circ}$. Note that through the shortcut method of neglecting the impedance of the null branch, the impedance is calculated as having the same value as that of the result from the wye-delta transformation approach. 


\section{COMPLEX CIRCUIT ANALYSIS EXAMPLE}

More complex circuits can also be addressed by the proposed method. As an example, consider complex network shown in Fig. 12. By inspection of the network, the circuit follows the balance relation (2). Therefore, the same procedure indicated in the othe examples is applied to this network and matched with the wye-delta approach.

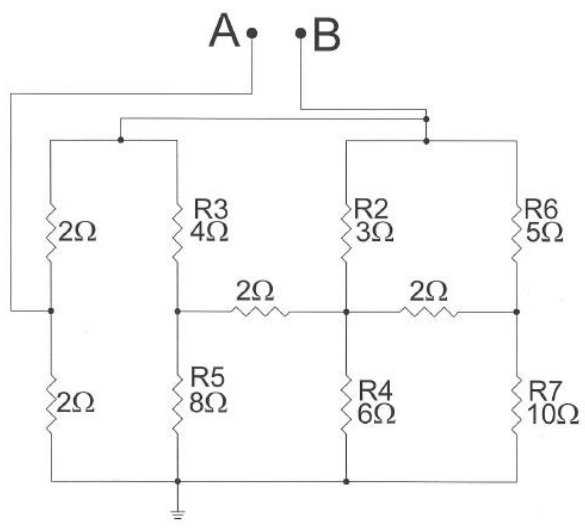

Fig 12: More Complex Circuit Example

By wye-delta approach the equivalent resistance between terminals A and B works out as 1.49 ohm. Two wye-delta transformatations and numerous series-parallel simplifications require lengthy time-consuming effort. However, since by inspection the balance equation (2) holds, the circuit of Fig. 12 can be redrawn as shown in Fig. 13.

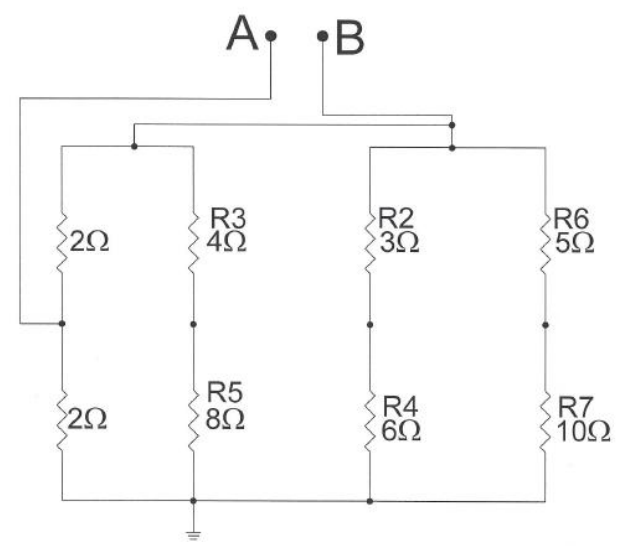

Fig 13: Simplified Circuit

Hence, by inspection the equivalent resistance between terminals $A$ and $B$ can be deduced as: $R(A B)=\{[(15\|9\| 12)+$ 2] $\| 2\}=1.49 \mathrm{ohm}$. Note that this result is same as for the circuit of Fig. 12, but the underlying computational task is highly reduced.

\section{CONCLUSION}

A shortcut method is indicated to find equivalent resistance/impedance of complex networks whenever certain balance conditions are satisfied. It is possible to see such balance conditions by simple inspection. If these balance conditions hold, then the circuit is simplified and the resistance/impedance in a null branch is assumed negligible. Thus, in summary, the present method allows a simplified approach to determine the equivalent resistance/impedance of complex $\mathrm{dc} / \mathrm{ac}$ circuits. The procedure is far more simpler than the wye-delta method. The present study is an extended and generalized method due to [1] with more and exhaustive application potentials.

\section{REFERENCES}

[1] Aneja, K. and Kumar, V. 2012. A New Simplified Approach to Find the Equivalent Resistance of Any Complex Equal Resistive Network. International Journal of Computers \& Technology, Vol. 3, No. 1.

[2] De Groff, D. 1999. Circuit Analysis, BarCharts, Inc., Boca Raton, FI. USA. 


\section{Author' biography with}

\section{Photo}

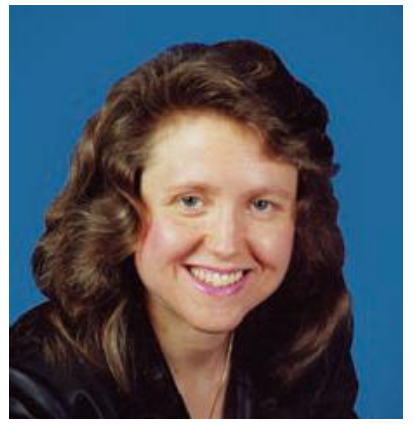

Dolores De Groff, Ph.D., is Associate Professor of Civil, Environmental, and Geomatics Engineering at Florida Atlantic University in Boca Raton.

She received her Ph.D. in Electrical Engineering from Florida Atlantic University in 1993. She has received several teaching awards from the same institution, coauthored a book in the neural network area and authored/coauthored other journal articles and conference papers. 